\title{
Video Article \\ Transplantation of Chemogenetically Engineered Cortical Interneuron Progenitors into Early Postnatal Mouse Brains
}

\author{
Myrto Denaxa ${ }^{1,2}$, Guilherme Neves ${ }^{3}$, Juan Burrone ${ }^{3}$, Vassilis Pachnis ${ }^{1}$ \\ ${ }^{1}$ Nervous System Development and Homeostasis Laboratory, The Francis Crick Institute \\ ${ }^{2}$ Neuroscience Centre, Biomedical Sciences Research Centre "Al. Fleming" \\ ${ }^{3}$ Centre for Developmental Neurobiology, King's College London
}

Correspondence to: Myrto Denaxa at denaxa@fleming.gr

URL: https://www.jove.com/video/59568

DOI: doi:10.3791/59568

Keywords: Neuroscience, Issue 150, cortical interneurons, ganglionic eminence, brain slice electroporation, intracranial injections, designer receptors exclusively activated by designer drugs (DREADD)

Date Published: 8/26/2019

Citation: Denaxa, M., Neves, G., Burrone, J., Pachnis, V. Transplantation of Chemogenetically Engineered Cortical Interneuron Progenitors into Early Postnatal Mouse Brains. J. Vis. Exp. (150), e59568, doi:10.3791/59568 (2019).

\section{Abstract}

Neuronal development is regulated by a complex combination of environmental and genetic factors. Assessing the relative contribution of each component is a complicated task, which is particularly difficult in regards to the development of $y$-aminobutyric acid (GABA)ergic cortical interneurons (Cls). Cls are the main inhibitory neurons in the cerebral cortex, and they play key roles in neuronal networks, by regulating both the activity of individual pyramidal neurons, as well as the oscillatory behavior of neuronal ensembles. They are generated in transient embryonic structures (medial and caudal ganglionic eminences - MGE and CGE) that are very difficult to efficiently target using in utero electroporation approaches. Interneuron progenitors migrate long distances during normal embryonic development, before they integrate in the cortical circuit. This remarkable ability to disperse and integrate into a developing network can be hijacked by transplanting embryonic interneuron precursors into early post-natal host cortices. Here, we present a protocol that allows genetic modification of embryonic interneuron progenitors using focal ex vivo electroporation. These engineered interneuron precursors are then transplanted into early post-natal host cortices, where they will mature into easily identifiable Cls. This protocol allows the use of multiple genetically encoded tools, or the ability to regulate the expression of specific genes in interneuron progenitors, in order to investigate the impact of either genetic or environmental variables on the maturation and integration of Cls.

\section{Video Link}

The video component of this article can be found at https://www.jove.com/video/59568/

\section{Introduction}

The function of neuronal networks relies in the existence of a balanced complement of excitatory projection neurons and inhibitory interneurons. Although cortical interneurons (Cls) only represent $20 \%$ of all neurons in the mammalian cortices, deficits in their number or function are thought to play a key part in the pathogenesis of neurodevelopmental disorders ${ }^{1,2}$. The study of $\mathrm{Cl}$ development is challenging because Cls are generated in transient embryonic structures that are hard to access, and they follow a long tangential migration before they reach the pallium and develop their mature anatomical and physiological properties ${ }^{3}$. Both genetic and environmental mechanisms are known that regulate $\mathrm{Cl}$ development ${ }^{4}$, but it has proven difficult to study the relative contribution of multiple factors.

Many insights in $\mathrm{Cl}$ development were obtained using in vitro culture systems after isolation of progenitors from the ganglionic eminences ${ }^{5,6}$. One of the great advantages of these methods is the potential to label and genetically modify the isolated progenitors and follow their differentiation in detail, to detect cell-autonomous changes. However, these methods are unable to offer information regarding the interactions between developing interneurons and an active network. We have adapted these protocols, by transplantation of the modified precursors into early postnatal cortex. Interneuron progenitors isolated from embryonic ganglionic eminences are able to survive, disperse and integrate into the host network upon transplantation into the cortex ${ }^{7,8}$. This method has been used to reduce the severity of epileptic seizures in genetic mouse models, and has been proposed as a possible new therapy for different neurodevelopmental disorders ${ }^{9,10}$. A previous protocol describes a procedure to transduce these precursors with viral vectors prior to transplantations ${ }^{11}$. The protocol we describe here also allows the genetic modification of interneurons, but does not require the creation of a viral vector, requiring only plasmid DNA, which greatly increases its flexibility. Some studies reported success in using in utero electroporation to genetically modify interneuron progenitors in the caudal ganglionic eminences (CGE) ${ }^{12}$, but this method has proven very difficult to reproduce.

In the representative results section, we illustrate the use of this method to express designer receptors exclusively activated by designer drugs $\left(\right.$ DREADDs $\left.{ }^{13}\right)$ in the transplanted Cls, a method we used in a recent publication ${ }^{14}$. We expressed $\mathrm{hM} 3 \mathrm{D}(\mathrm{Gq})$, an engineered receptor based on the human cholinergic receptor $\mathrm{CHRM} 3$, which does not affect neuronal function unless it binds its specific ligand clozapine-N-oxide (CNO). $\mathrm{CNO}$ administration selectively triggers activation of $\mathrm{hM} 3 \mathrm{D}(\mathrm{Gq})$ expressing cells. We used this method to show that cell autonomous and transient depolarization is sufficient to prevent apoptosis of Cls during development ${ }^{14}$. Combined with different genetically encoded tools, this 
protocol has the potential to up- or down-regulate gene expression, and visualize or manipulate cell activity during different stages of interneuron differentiation.

Protocol

Animals were bred and housed in accordance with the United Kingdom Animals (Scientific Procedures) Act (1986).

NOTE: For the generation of the pCAGGs-hM3D(Gq)-IRES-RFP construct, a Sall-Stul fragment, containing the hM3D(Gq) sequence, has been isolated from plasmid 50463 (Addgene), and inserted into the expression vector pCAGGs-RFP (gift from F. Guillemot) digested with Xhol-EcoRV.

\section{Preparation of Mouse Embryo Cortical Slices}

1. Sterilize lab equipment (e.g., stereo microscopes) and surfaces (e.g., benches) with an appropriate detergent solvent and $70 \%$ ethanol $(\mathrm{EtOH})$ solution in water $\left(\mathrm{H}_{2} \mathrm{O}\right)$.

2. Use dissection tools that have been autoclaved and keep them all the time in $100 \% \mathrm{EtOH}$.

3. Prepare three $20 \mathrm{~mL}$ aliquots of $4 \%$ low-gelling temperature agarose in $1 \times$ phosphate buffer solution (PBS), in $50 \mathrm{~mL}$ tubes, and keep them at $55^{\circ} \mathrm{C}$.

4. Sacrifice pregnant mice by cervical dislocation at 13.5 or 14.5 days of gestation (embryonic day E13.5-E14.5).

5. With a pair of dissection scissors, open the abdomen of the pregnant mice, remove the uterine horns (with the embryos) and place them in ice-cold Krebs solution (Table 1), in a $90 \mathrm{~mm}$ Petri dish.

6. With a pair of straight student fine forceps, open the amniotic sacs, remove the embryos and transfer them in fresh ice-cold Krebs solution, in a new $90 \mathrm{~mm}$ Petri dish. Dissect the mouse embryo brain (forebrain, midbrain, hindbrain) from the rest of the embryo body.

7. Holding the dissected brains from the hindbrain, transfer them in ice-cold Krebs solution in a new $90 \mathrm{~mm}$ Petri dish, and keep them on ice.

8. With a waterproof pen, draw a straight line at the outside surface, in the middle of the bottom part of six $35 \mathrm{~mm}$ Petri dishes.

9. Place one $4 \%$ low-gelling agarose/PBS $20 \mathrm{~mL}$ aliquot at $37^{\circ} \mathrm{C}$ for $5 \mathrm{~min}$. Immediately afterwards poor $10 \mathrm{~mL}$ in two $35 \mathrm{~mm}$ Petri dishes and embed the dissected brains.

NOTE: The olfactory bulbs should face downwards (bottom of the Petri dish). Embed 3-4 brains per Petri dish, align them in the straight line previously drawn. Leave 3-5 mm space between each two brains.

10. Repeat steps 1.8 and 1.9 until all dissected brains have been embedded.

11. Place the embedded brains at $4{ }^{\circ} \mathrm{C}$, in order for the agarose to solidify, and subsequently carve the three brains into a single block of appropriate size and orientation.

NOTE: Leave approximately $3 \mathrm{~mm}$ around the edges of the brain samples. Change the orientation of the brains, with the olfactory bulbs on the top.

12. Glue the block on the surface of a microtome base and using a surgical blade cut all the way through the bottom of the block, between each two brains, in order to create 3 independent blocks (each mini block containing one brain).

13. Section the blocks, in ice-cold Krebs solution, into $250 \mu \mathrm{m}$ thick slices, using a vibrating blade microtome.

14. With a bended flat-ended micro-spatula collect only the slices containing the medial or caudal ganglionic eminences (MGE or CGE; Figure 1) and individually transfer them onto filter membranes $(13 \mu \mathrm{m}$ diameter, $8.0 \mu \mathrm{m}$ pore size), floating on minimal essential medium (MEM, Table 1) in polystyrene center-well organ culture dishes $(60 \mathrm{~mm} \times 15 \mathrm{~mm})$.

15. Place the dishes in a $\mathrm{CO}_{2}$ tissue culture incubator, at $37^{\circ} \mathrm{C}$, for $1 \mathrm{~h}$, and prepare for the focal electroporation.

\section{Acute Brain Slice Electroporation}

1. Before beginning the electroporation procedure, prepare $50 \mathrm{~mL}$ of $1 \%$ agarose gel in a $100 \mathrm{~mm}$ Petri dish. Leave the agarose gel to solidify at room temperature $(\mathrm{RT})$ for approximately $30 \mathrm{~min}$.

2. Prepare tiny agarose columns (1 $\mathrm{mm}$ diameter and $10 \mathrm{~mm}$ length), punched with a glass pipette (225 mm length; $2 \mathrm{~mL}$ capacity) and transfer them in ice-cold Krebs solutions.

NOTE: A rubber dropper bulb suitable for $2 \mathrm{~mL}$ pipettes is attached to the pipette, and by pressing it the column can be released from the glass pipette to the Krebs solution.

3. With a surgical blade, cut small agarose blocks of two sizes: a small one that will fit on the surface of the electrode (see below) and a bigger one, which will be used as a base for performing the focal DNA injections into the brain slices. Transfer the agarose blocks in ice-cold Krebs solution as well.

4. Prepare the set-up for the focal injections and the acute electroporation (Figure 2).

NOTE: For the injections, the following equipment is needed: 1) a bright field dissection stereo-microscope, 2) a pneumatic pico-pump injector, 3) a micromanipulator, 4) a magnetic stand, and 5) a steel base plate. For the acute slice electroporation, the following equipment is needed: 1) one square platinum $10 \mathrm{~mm}$ Petri dish electrode, 2) one square platinum $10 \mathrm{~mm}$ cover electrode, 3) a micromanipulator, and 4) an electroporator.

5. Focal DNA injections

1. Prepare a DNA mixture of expression vectors: pCAGGs-IRES-GFP (control vector) + pCAGGs-hM3D(Gq)-IRES-RFP, at a concentration of $1 \mu \mathrm{g} / \mu \mathrm{L}$ for each vector and add fast green solution (stock $25 \mathrm{mg} / \mathrm{mL}$ ) in a 1/10 dilution.

2. Fill a pulled glass micropipette $(0.5 \mathrm{~mm}$ inner diameter and $1 \mathrm{~mm}$ outer diameter) with $10 \mu \mathrm{L}$ of the DNA mixture and inject small amounts (on the 25-50 nL range) into the selected region (MGE/CGE) of the slice (Figure 1 and Figure 2).

6. Acute electroporation

NOTE: The electroporation should be performed immediately after the focal DNA injection.

1. Place the small agarose block on the Petri dish electrode and attach the agarose column to the mobile cover electrode with the help of a flat-ended micro-spatula. 
2. Transfer the slice with its supporting membrane onto the agarose block and place the top electrode with the agarose column on top of the selected region (MGE/CGE) of the slice.

NOTE: Charging voltages of $125 \mathrm{~V}$ (two pulses of $5 \mathrm{~ms}$ each, interval $500 \mathrm{~ms}$ ) will yield a successful electroporation (Figure 3).

7. After the electroporation, place the slice with its supporting membrane into the dish and transfer the dish in a $\mathrm{CO}_{2}$ tissue culture incubator, at $37^{\circ} \mathrm{C}$.

8. After $1 \mathrm{~h}$, exchange the MEM medium for a basic medium appropriate for primary neuronal cultures (neuron basic medium; Table 1) and incubate the slices overnight, for approximately $18-24 \mathrm{~h}$.

\section{Preparation of Cell-grafts}

1. Check the efficiency of the electroporation in all slices. Select only the slices where an acceptable number of fluorescent cells is observed (Figure 3).

NOTE: Electroporate approximately 30 slices/per experiment in order to acquire the appropriate number of cells for grafting (see below).

2. Dissect the selected region (MGE/CGE) from each slice and cut the tissue into small pieces in ice cold Krebs solution, under a fluorescent dissection stereo-microscope.

3. Meanwhile, place a $1.5 \mathrm{~mL}$ tube with $900 \mu \mathrm{L}$ neuron basic medium in a water bath at $37^{\circ} \mathrm{C}$.

4. Transfer the tissue pieces with a P1000 micropipettor to a $1.5 \mathrm{~mL}$ tube containing $500 \mu \mathrm{L}$ of L15/DNase medium prepared by adding $100 \mu \mathrm{L}$ of $1 \mathrm{mg} / \mathrm{mL}$ DNase stock in DMEM/F12 medium into $900 \mu \mathrm{L}$ of L15 medium. Wash the tissue pieces by tapping.

5. Add $100 \mu \mathrm{L}$ of DNase (stock $1 \mathrm{mg} / \mathrm{mL}$ in DMEM/F12 medium) into the $900 \mu \mathrm{L}$ neuron basic medium.

6. Discard the L15/DNase medium and resuspend the tissue pieces in $200 \mu \mathrm{L}$ neuron basic/DNase medium prepared in step 3.5 .

7. Set a P200 micropipettor to $180 \mu \mathrm{L}$ and mechanically dissociate the tissue pieces by pipetting up and down gently, 20-30 times, until a smooth and "creamy" suspension is obtained.

8. Add $200 \mu \mathrm{L}$ of neuron basic/DNase medium (final total volume $400 \mu \mathrm{L}$ ) and resuspend.

9. Take a $4 \mu \mathrm{L}$ aliquot of cells, dilute appropriately and mount on a haemocytometer.

10. Under a bright field microscope, check the efficiency of the dissociation and count the number of cells. NOTE: If the dissociation is successful, bright (alive) single cells and not cell-aggregates will be observed.

11. Centrifuge cell suspension from step 3.8, at $1,000 \mathrm{rpm}$, at RT, for $5 \mathrm{~min}$, and subsequently, remove the supernatant from the tube and add the appropriate L15/DNase medium (usually 5-7 $\mu \mathrm{L}$ ) so that the final concentration of cells will be $8 \times 10^{5}-1.2 \times 10^{6}$ cells $/ \mu \mathrm{L}$.

NOTE: During the resuspension, it is extremely important to avoid air bubbles.

12. Place the cell aliquot on ice and have additional L15/DNase medium for the injections.

\section{Intracranial Injections}

NOTE: The following procedures take place in a procedure room within the Animal House Facility. Since the cells will be injected directly to the brain of newborn pups without exposing the brain, aseptic conditions are kept by sterilizing the working space with $70 \% \mathrm{EtOH}$ solution and using autoclaved glass needles. The following equipment is needed for the intracranial injections: 1) a bright field dissection stereo-microscope, 2) a micro-injector, and 3) a heating pad for mouse recovery.

1. Prepare a glass needle by pulling needles according to the manufacturer's recommendations. Glass needles with an $80 \mu \mathrm{m}$ outer diameter, $40 \mu \mathrm{m}$ inner diameter, and a $30^{\circ}$ bevel are used in this protocol.

NOTE: As mentioned above, needles should be autoclaved before use.

2. Backfill manually the needle with biologically inert oil using a $30 \mathrm{G}, 2$ inch needle and a syringe.

3. Assemble and insert the needle to the injector unit according to the manufacturer's instructions.

4. Determine the injection settings at maximum volume $(69 \mathrm{~nL})$ and a relative slow rate $(23 \mathrm{~nL} / \mathrm{s})$.

5. Empty the needle until the plunger is fully extended.

6. Fill the needle.

1. Cut a small piece from a grafting tape and place it under a bright field dissection stereo-microscope. With a P10 micropipette, transfer 5 $\mu \mathrm{L}$ from the sample (cell aliquot) onto the tape, so that a spherical drop is formed.

2. Place the tip of the needle into the sample and fill the needle (the plunger retracts and draws the sample with it).

NOTE: Since the sample should be quite viscous, fill the needle in small steps so that the sample will equilibrate, and at a slow rate that prevents bubbles from forming. The sample should be smooth and homogeneous within the needle. If the sample is too viscous and it is not possible to fill the needle, add as much L15/DNase medium as it is required to obtain the correct viscosity. Nevertheless, this will change the concentration of the sample, and ideally should be avoided.

7. Anaesthetize new born pups (postnatal day 0-2 [P0-P2]) on ice for 2-5 min. NOTE: Make sure that the pup is not moving.

8. Place the anaesthetized pup under the bright field dissection stereo-microscope.

9. Perform 3-4 injections of $69 \mathrm{~nL}$ each in each hemisphere.

NOTE: The injection sites are located approximately $1 \mathrm{~mm}$ lateral to the midline, and between $1 \mathrm{~mm}$ caudal to bregma and $1 \mathrm{~mm}$ rostral to the interaural line. The tip of the needle should be placed approximately $1 \mathrm{~mm}$ deep to the pial surface. After each injection, the needle is left in place for approximately $30 \mathrm{~s}$ and withdrawn in periods.

10. Immediately after the injections, place the pup on a heating pad with the heating at its lowest setting $\left(37^{\circ} \mathrm{C}\right)$. When the pup recovers transfer it to the cage with its mother.

NOTE: Never leave the mother with no pups in the cage. The whole procedure (from removing the pup from its littermates, until it is returned) should last less than $10 \mathrm{~min}$. 


\section{Clozapine-N-oxide Injections}

1. Prepare the DREADD ligand, CNO stock solution by diluting $1 \mathrm{mg}$ of $\mathrm{CNO}$ in $50 \mu \mathrm{L}$ of dimethyl sulfoxide (DMSO) until the solution is translucent. Top-up to $10 \mathrm{~mL}$ with saline so that the final concentration of CNO is $0.1 \mathrm{mg} / \mathrm{mL}$.

NOTE: DMSO is toxic. Avoid using it to a concentration higher than $0.1 \%$. Use as a control a saline solution containing the same DMSO concentration as the CNO containing solution.

2. From postnatal day 14 and for 3 constitutively days (P14-P16), in each mouse perform two intraperitoneal (I.P.) injections of CNO (CNO concentration: $1 \mathrm{mg} / \mathrm{kg}$ ) or $0.05 \%$ DMSO in saline, per day, $12 \mathrm{~h}$ apart.

3. On the last day (P17), perform a single injection and sacrifice mice by cervical dislocation within a time window of $30 \mathrm{~min}-1 \mathrm{~h}$.

\section{Representative Results}

Using the procedure presented here, we tested whether the survival of cortical interneurons during early postnatal stages is regulated by activity in a cell autonomous manner. We performed 3 brain slice electroporation experiments (12-16 embryos [E14.5 embryos] per experiment) with the pCAGGs-IRES-GFP (control) and pCAGGs-hM3D(Gq)-IRES-RFP expression vectors, at a concentration of $1 \mu \mathrm{g} / \mu \mathrm{L}$ for each construct. In our electroporation experiments, only a fraction (approximately 50\%; Figure 3) of the GFP neurons co-expressed $h M 3 D(G q)\left(R F P{ }^{+}\right)$and therefore the GFP ${ }^{+}$RFP $^{-}$population served as an internal control for the effect of DREADD ligands. Transfected cortical embryonic interneurons were mechanically dissociated and the resulting cell suspension $\left(8 \times 10^{5}\right.$ cells/ $\left.\mu \mathrm{L}\right)$ grafted in the cortex of P0-P1 wild type mice. We had performed 6 injections per brain. In each experiment, a minimum of 6 new born pups were injected. Administration of CNO selectively increased the activity of transfected $\mathrm{RFP}^{+}$cells, as demonstrated by the expression of the activity-dependent protein cFos (Figure 4). CNO treatment according to the described protocol (administer twice daily P14-P17) resulted in an increase in the proportion of GFP ${ }^{+}$RFP $^{+}$relative to GFP ${ }^{+}$RFP $^{-}$cells, in comparison to vehicle ( $0.5 \%$ DMSO in saline) administered littermates (Figure 5).
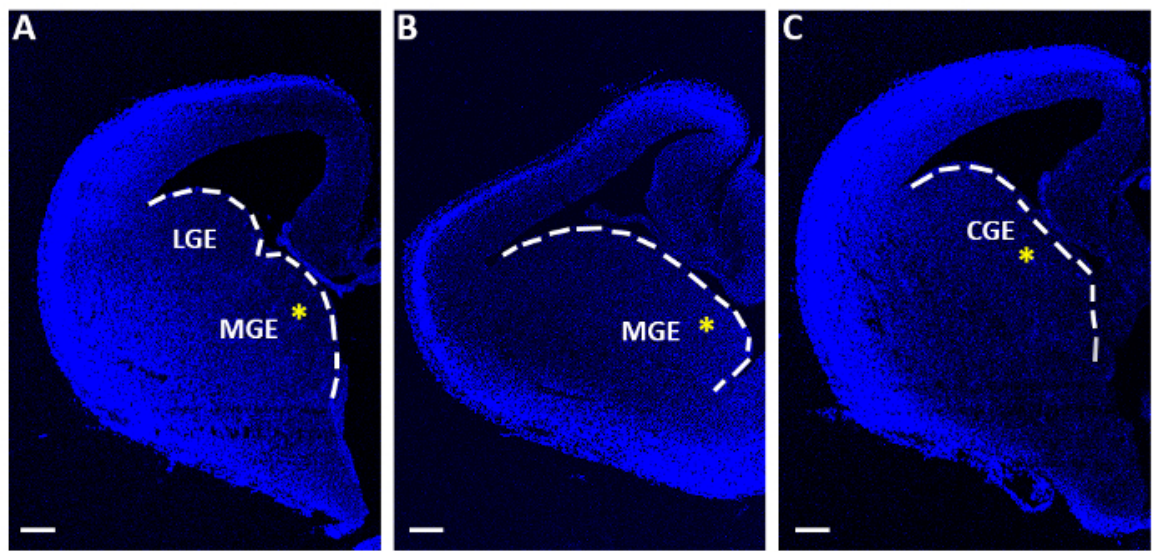

Figure 1: Representative telencephalic slices used for acute electroporation experiments. (A-C) Telencephalic slices obtained at three distinct sequential rostro-caudal levels, stained with 4',6-diamidino-2-phenylindole (DAPI). LGE: lateral ganglionic eminence; MGE: medial ganglionic eminence; CGE: caudal ganglionic eminence. Scale bars $=200 \mu \mathrm{m}$. The yellow asterisks indicate the electroporation site in each slice. The white line marks the edge of the ganglionic eminence. Please click here to view a larger version of this figure. 


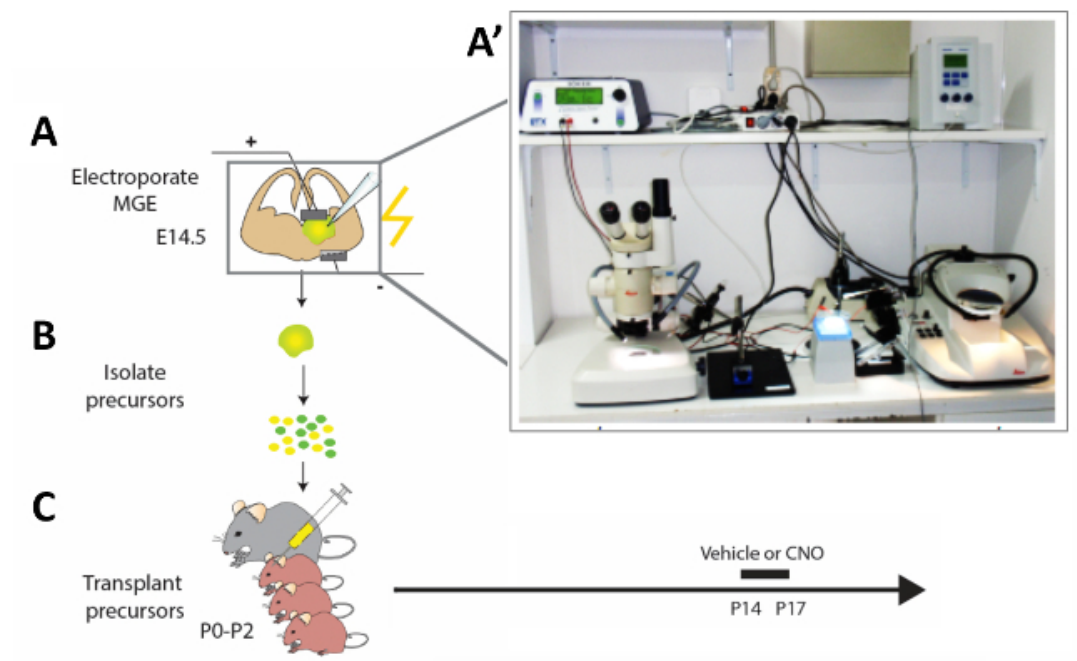

Figure 2: Schematic representation of the experimental workflow. (A) Mouse brain slices are electroporated with appropriate constructs, and $(\mathrm{B})$ after $12 \mathrm{~h}$ modified cortical interneuron $(\mathrm{Cl})$ precursors are isolated and $(\mathbf{C})$ transplanted in the pallium of newborn mouse pups (P0-P2). In order to modify the activity of immature Cls, P14 pups that had received cell transplantations were injected with CNO or vehicle for four constitutive days according to the presented protocol. (A') Photograph of the acute mouse brain slice electroporation set-up. Please click here to view a larger version of this figure.
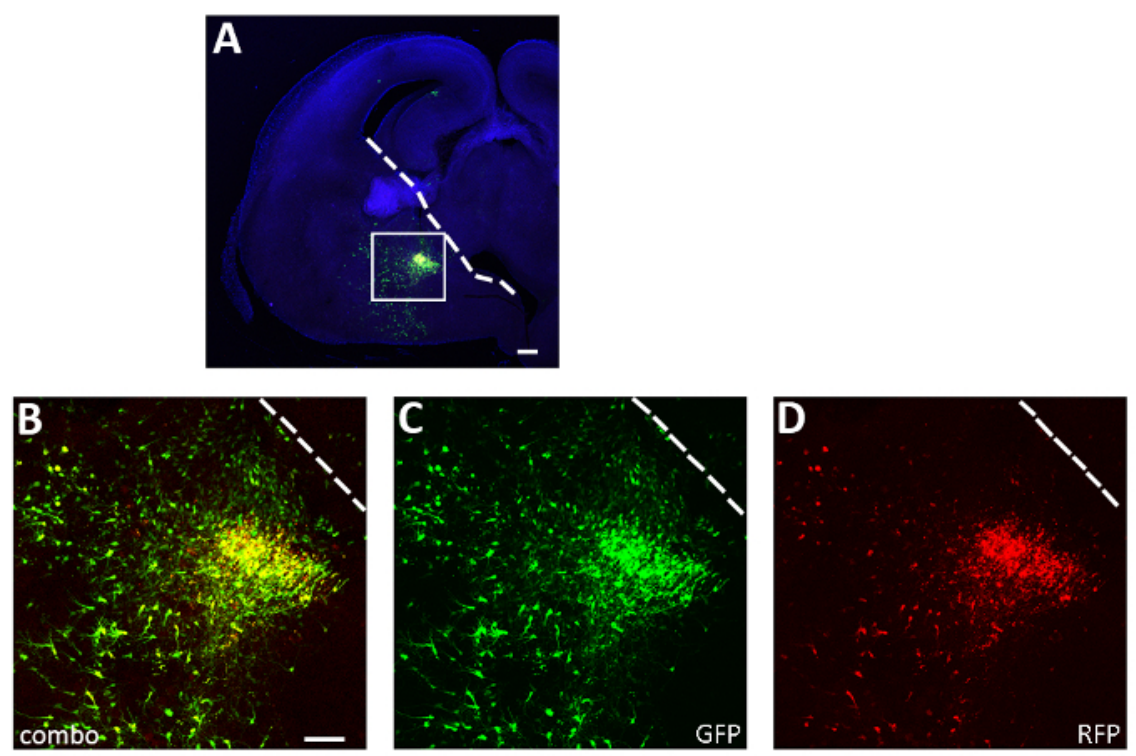

Figure 3: Representative successful acute slice electroporation experiment. (A) Representative coronal section from an $\mathrm{E} 14.5$ embryo brain transfected in the CGE with both pCAGGs-IRES-GFP (GFP) and pCAGGs-hM3D(Gq)-IRES-RFP (RFP) plasmids and cultured for $12 \mathrm{~h}$. The section has been immunostained for GFP $(A, B, C)$ and $\operatorname{RFP}(A, B, D)$. The boxed area in panel $A$ is magnified to show the expression of both fluorescent reporters (B), GFP (C) and RFP only (D). The white line marks the edge of the ganglionic eminence. B-D: same photo, different channels or combination of the two different channels. Scale bars $=200 \mu \mathrm{m}(A), 100 \mu \mathrm{m}(B-D)$. This figure has been modified from Denaxa et al. ${ }^{14}$. Please click here to view a larger version of this figure. 

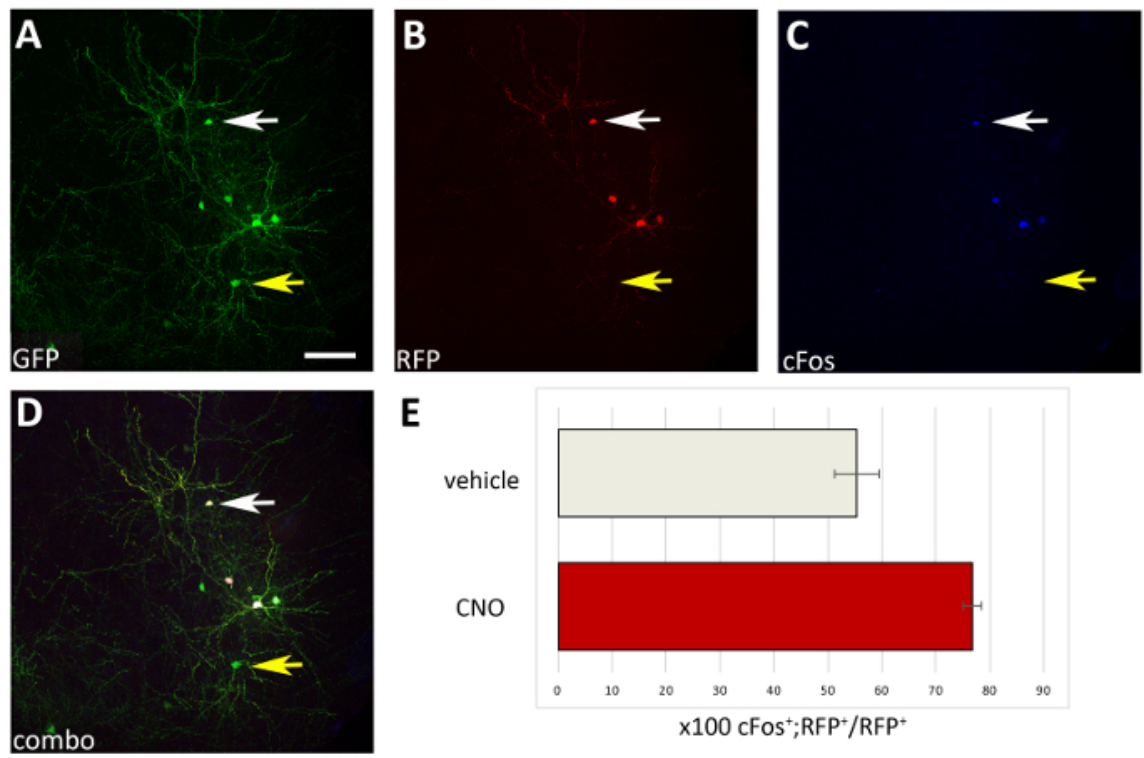

E

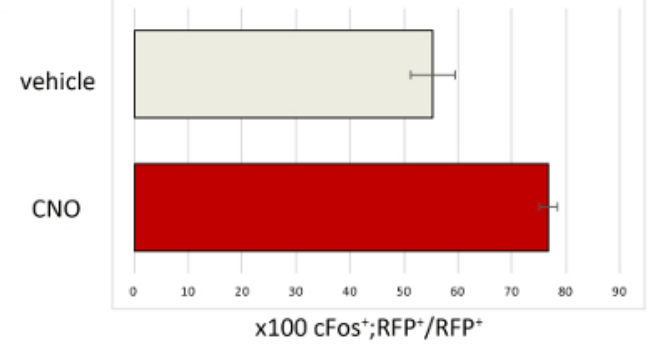

Figure 4: Cell autonomous increase in the activity of $M 3 D(G q)$-expressing transplanted Cls upon CNO administration. (A-D)

Representative confocal images of a coronal section of a P17 mouse transplanted at P1 with CI precursors transfected with both pCAGGs-IRESGFP (GFP) and pCAGGs-hM3D(Gq)-IRES-RFP (RFP) plasmids and treated with CNO. The section has been immunostained for GFP (A), RFP (B), and cFos (C). (D) The combined image of A, B and C immunofluorescence (combo). Note that only Cls co-expressing both plasmids (white arrows in A-D) are also cFos ${ }^{+}$compared to Cls expressing only the control-GFP plasmid (yellow arrows in A-D). (E) Quantification of cFos ${ }^{+} R F P^{+}$ cells found in the pallium of P17 mice transplanted at P1 (normalized to the total RFP ${ }^{+}$population) and treated with vehicle or CNO (N $=2$ ). A-D: same photo, different channels, or combination of the three different channels. Scale bars $=50 \mu \mathrm{m}$. Please click here to view a larger version of this figure.
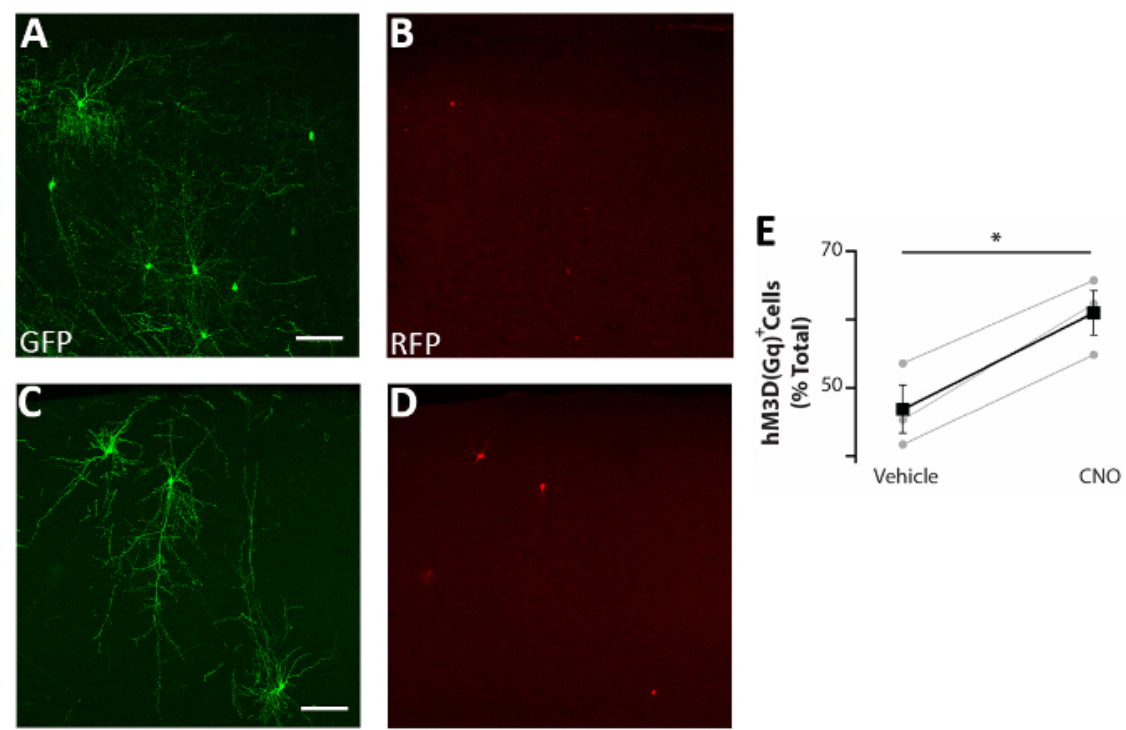

Figure 5: Cell autonomous increase in the activity of Cls enhances survival. (A-D) Representative confocal images of somatosensory cortex coronal slices of P17 mice transplanted at P0-P2 with Cl precursors transfected with both pCAGGs-IRES-GFP (GFP) and pCAGGshM3D(Gq)-IRES-RFP (RFP) plasmids and treated with vehicle (A-B) or CNO (C-D). (E) Quantification of RFP ${ }^{+}$cells found in the forebrain of P17 mice transplanted at P0-P2 (normalized to the total $\mathrm{GFP}^{+}$population). $\mathrm{RFP}^{+}$(vehicle) $=47 \% \pm 3 \%, \mathrm{CNO}=61 \% \pm 3 \%, \mathrm{p}=0.01$, Student's paired sample $t$ test, $n=3$ vehicle and $3 \mathrm{CNO}$, a minimum of 150 cells counted per brain. A and B: same photo, different channels. C and D: same photo, different channels. Scale bars $=50 \mu \mathrm{m}$. This figure has been modified from Denaxa et al. ${ }^{14}$. Please click here to view a larger version of this figure. 


\begin{tabular}{|c|c|c|}
\hline KREBS & Chemicals & Concetration \\
\hline \multicolumn{3}{|c|}{$10 x$ KREBS (stock) } \\
\hline \multirow[t]{6}{*}{$500 \mathrm{~mL}$} & $\mathrm{NaCl}$ & $1.26 \mathrm{M}$ \\
\hline & $\mathrm{KCl}$ & $25 \mathrm{mM}$ \\
\hline & $\mathrm{NaH}_{2} \mathrm{PO}_{4}$ & $12 \mathrm{mM}$ \\
\hline & $\mathrm{MgCl}_{2}$ & $12 \mathrm{mM}$ \\
\hline & $\mathrm{CaCl}_{2}$ & $21 \mathrm{mM}$ \\
\hline & $\mathrm{dH}_{2} \mathrm{O}$ (sterile) & $500 \mathrm{~mL}$ \\
\hline \multicolumn{3}{|c|}{ 1x KREBS } \\
\hline \multirow[t]{4}{*}{$500 \mathrm{~mL}$} & 10x KREBS & $50 \mathrm{~mL}$ \\
\hline & Glucose & $0.99 \mathrm{gr}$ \\
\hline & $\mathrm{NaHCO}_{3}$ & $1.05 \mathrm{gr}$ \\
\hline & $\mathrm{dH}_{3} \mathrm{O}$ (sterile) & $450 \mathrm{~mL}$ \\
\hline \multicolumn{3}{|l|}{ MEM } \\
\hline \multirow[t]{4}{*}{$50 \mathrm{~mL}$} & MEM with glutamine & $43.5 \mathrm{~mL}$ \\
\hline & FCS (Fetal Bovine Serum) & $5 \mathrm{~mL}$ \\
\hline & $25 \%$ Glucose in $\mathrm{dH}_{2} \mathrm{O}$ (sterile) & $1 \mathrm{~mL}$ \\
\hline & 100x Penicillin/Streptomycin (Pen/Strep) & $0.5 \mathrm{~mL}$ \\
\hline \multicolumn{3}{|c|}{\begin{tabular}{|l|} 
Neuron Basic Medium \\
\end{tabular}} \\
\hline \multirow[t]{5}{*}{$50 \mathrm{~mL}$} & Neuron Basic Medium & $47 \mathrm{~mL}$ \\
\hline & B-27 supplement & $1 \mathrm{~mL}$ \\
\hline & $25 \%$ Glucose in $\mathrm{dH}_{2} \mathrm{O}$ (sterile) & $1 \mathrm{~mL}$ \\
\hline & 100x Pen/Strep & $0.5 \mathrm{~mL}$ \\
\hline & 100x Glutamine & $0.5 \mathrm{~mL}$ \\
\hline
\end{tabular}

Table 1: Additional information concerning media used in this protocol.

\section{Discussion}

Here we describe a widely accessible methodology to genetically modify the activity of $\mathrm{Cl}$ precursors to study the impact of intrinsic activity on $\mathrm{Cl}$ maturation, and/or the effect of activity modulated Cls on the assembly/function of the integrated cortical circuits.

In the past, several labs, including ours, had performed in utero electroporation experiments in order to genetically modify projection neurons ${ }^{6}$. However, in utero electroporation into ganglionic eminences that include $\mathrm{Cl}$ progenitors is very difficult, due to electrical conduction path problems. In order to solve this problem, a small number of labs are performing ultrasound guided injections followed by electroporation, which is a demanding technique that requires expensive equipment. This protocol provides an alternative to these methodologies which is accessible to the majority of the scientific community.

One of the most challenging aspect of this protocol is to maximize the number of cells that survive in the host cortex to mature stages, when phenotypic analysis is usually performed (very dependent on the experiment design, but typically older than P17). There are three key steps that the investigator should pay attention: (1) The efficiency of the electroporation. This can be maximized by ensuring the purity of DNA plasmids. Only high-quality DNA plasmids (an $\mathrm{A}_{260} / \mathrm{A}_{280}$ ratio of 1.9-2.0) should be used for this procedure. We obtain such high-quality DNA preparations by employing cesium chloride DNA purification. Another crucial factor is the promoter that drives the expression of the gene of interest. We found that the pCAGGs vector, which consists of the chicken b-actin promoter, is extremely powerful and can dramatically increase the electroporation efficiency. (2) The number of starting donor embryos. It is important to make sure that a large number (12-16) of embryos of the same stage are electroporated. This number can be increased, if more experimenters are performing embryo dissections and sectioning together, as it important that embryonic cortical slices are obtained, electroporated and transferred to the incubator as soon as possible. (3) It is important to make sure that a great number of cells are injected in each pup to ensure a high chance of transplanted cell survival until mature stages. In addition, this will dramatically improve the likelihood of successful transplants since low density cell preparations will result in uneven mixing of the cells with the medium, which will produce significant variability in the transplanted brains ${ }^{15}$.

The protocol described here was tailored for investigating the role of activity in regulating $\mathrm{Cl}$ survival in a cell-autonomous manner. The P14-P17 time window for performing the CNO injections was specifically chosen according to published data, which show that the peak of transplanted $\mathrm{Cl}$ progenitors' cell death occurs during this period ${ }^{16}$. Therefore, this time frame or the frequency of CNO injections might not hold true for other cell types or brain regions, and the investigator should adjust these parameters according to the specific experimental purposes. Finally, the methodology described here for the intracranial injections of Cls is only feasible for P0-P5 pups (depending also on the mouse line background). In principle, any injections over P5 will require thinning or removing of the skull ${ }^{15}$.

One of the key advantages of this protocol is the ability to use new genetically encoded tools to visualize or manipulate the activity of Cls during different stages of differentiation as they integrate into a developing network. With the pace of discovery of new genetically encoded voltage and calcium sensors, as well as new chemogenetic and optogenetic tools, this protocol allows researchers to use them within weeks of release into plasmid repositories, such as Addgene.

\section{Disclosures}

The authors have nothing to disclose.

\section{Acknowledgments}

This work was supported by an ERC Starter Grant (282047), a Wellcome Trust Investigator Award (095589/Z/11/Z), an FP7 EC DESIRE grant, and a Lister Institute Prize to JB. Work in V.P.'s laboratory is supported by the BBSRC (BB/L022974/1), the UK Medical Research Council 
(MRC), and the Francis Crick Institute (which receives funding from the MRC, Cancer Research UK, and the Wellcome Trust). The research in M.D. lab was made possible through the grant from the Stavros Niarchos Foundation to the B.S.R.C. "Alexander Fleming", as part of the Foundation's initiative to support the Greek research.

\section{References}

1. Marin, O. Interneuron dysfunction in psychiatric disorders. Nature Review Neuroscience. 13 (2), 107-120, (2012).

2. Glausier, J.R., Lewis, D.A. GABA and schizophrenia: Where we stand and where we need to go. Schizophrenia Research. 181, 2-3, (2017).

3. Fishell, G., Rudy, B. Mechanisms of inhibition within the telencephalon: "where the wild things are". Annual Review Neuroscience. 34, 535-567, (2011).

4. Wamsley, B., Fishell, G. Genetic and activity-dependent mechanisms underlying interneuron diversity. Nature Review Neuroscience. 18 (5), 299-309, (2017).

5. Xu, Q., Cobos, I., De La Cruz, E., Rubenstein, J.L., Anderson, S.A. Origins of cortical interneuron subtypes. Journal of Neuroscience. 24 (11), 2612-2622, (2004).

6. Denaxa, M., et al. Maturation-promoting activity of SATB1 in MGE-derived cortical interneurons. Cell Reports. 2 (5), 1351-1362, (2012).

7. Wichterle, H., Garcia-Verdugo, J.M., Herrera, D.G., Alvarez-Buylla, A. Young neurons from medial ganglionic eminence disperse in adult and embryonic brain. Nature Neuroscience. 2 (5), 461-466, (1999).

8. Alvarez-Dolado, M., et al. Cortical inhibition modified by embryonic neural precursors grafted into the postnatal brain. Journal of Neuroscience. 26 (28), 7380-7389, (2006).

9. Baraban, S.C., et al. Reduction of seizures by transplantation of cortical GABAergic interneuron precursors into Kv1.1 mutant mice. Proccedings of the National Academy of Sciences of the United States of America. 106 (36), 15472-15477, (2009).

10. Southwell, D.G., et al. Interneurons from embryonic development to cell-based therapy. Science. 344 (6180), 1240622, (2014).

11. Vogt, D., et al. Viral-mediated Labeling and Transplantation of Medial Ganglionic Eminence (MGE) Cells for In Vivo Studies. Journal of Visualized Experiments. (98), (2015).

12. De Marco Garcia, N.V., Karayannis, T., Fishell, G. Neuronal activity is required for the development of specific cortical interneuron subtypes. Nature. 472 (7343), 351-355, (2011).

13. Urban, D.J. Roth, B.L. DREADDs (designer receptors exclusively activated by designer drugs): chemogenetic tools with therapeutic utility. Annual Review of Pharmacology and Toxicology. 55, 399-417, (2015).

14. Denaxa, M., et al. Modulation of Apoptosis Controls Inhibitory Interneuron Number in the Cortex. Cell Reports. 22 (7), 1710-1721, (2018).

15. Quatrocolo, G. et al. Homochronic Transplatation of Interneuron Precursors into Early Postnatal Mouse Brains. Journal of Visualized Experiments. (136), (2018).

16. Southwell, D.G. et al. Intrinsically determined cell death of developning cortical interneurons. Nature. 491 (7422), 103-113, (2012). 